\title{
Spectral Signature Analysis - BIST for RF Front-Ends
}

\author{
D. Lupea, U. Pursche, and H.-J. Jentschel \\ Technische Universität Dresden, Institut für Verkehrsinformationssysteme, Mommsenstr. 13, D-01062 Dresden
}

\begin{abstract}
In this paper, the Spectral Signature Analysis is presented as a concept for an integrable self-test system (Built-In Self-Test - BIST) for RF front-ends is presented. It is based on modelling the whole RF front-end (transmitter and receiver) on system level, on generating of a Spectral Signature and of evaluating of the Signature Response. Because of using multi-carrier signal as the test signature, the concept is especially useful for tests of linearity and frequency response of front-ends. Due to the presented method of signature response evaluation, this concept can be used for Built-In Self-Correction (BISC) at critical building blocks.
\end{abstract}

\section{Introduction}

Presently, a rapidly growing integration density and more complex design structures of systems in mobile telecommunications are the characteristics of microelectronics. Taking into account the enormous cost pressure, the reduction of costs for test overhead is an important item. For that purpose Built-In-Self-Test (BIST) is applied as a method of integrating suitable test structures on the chip. A special challenge is the application of BIST in the RF- and mixed signal domain. In contrast to the test of digital systems, analog systems have only a few inputs and outputs and their internal states exhibit low time constants. From that follows, that a test with a high coverage is possible with small effort. Indeed, the more complicated task is the generation of the test stimulus. Therefore, this paper is concerned mainly with the problem of test stimulus generation. The object of investigations is the RF front-end. The text is structured as follows. Section 2 shortly outlines the selection of a test strategy. Section 3 describes a theoretical approach to evaluate the test signature response. In Sect. 4 our proposal for an universal front-end BIST is outlined. Section 5 presents a simulation environment for generating and analysing signature test

Correspondence to: U. Pursche

(pursche@vini.vkw.tu-dresden.de) stimuli and for modelling different devices under test with adjustable non-idealities. Conclusions are drawn in Sect. 6.

\section{Test strategy}

Possible strategies (Bushnell and Agrawal, 2001) to implement BIST for an RF front-end can be divided in two categories. Well known from the literature is the separate test of different single building blocks of the RF front-end (Huang et al., 2000). In this case every block corresponds to a device under test (DUT) and a special test signature will be formed according to the DUT's requirements. In addition, an appropriate processing of the DUT's signature response is necessary. The advantage of this principle is a high test coverage due to the usage of a special test stimulus that take into account all test conditions of the specific DUT. The test signature can be specially designed. The main disadvantage is the high test overhead. It results from the necessity to design a special test set-up for each block to be tested. Moreover, it will be assumed that all building blocks used to generate the test stimulus and to convert the response are ideal. Therefore any imperfections and failures of these building blocks will cause corresponding failures in the test

The second strategy (Hafed et al., 2000; Hafed and Roberts, 2000) consists in testing the whole transceiver frontend as a complete system (Fig. 1). In this case, the DUT's structure corresponds to a chain of building blocks of a transmitter and a receiver which are connected in a loop at the antennas. This principle is known from point-to-point radios as loopback technique (Nowakowski et al., 2001). The test signature will be injected in the transmitter's baseband interface and the signature response of the DUT will be evaluated on the receiver's baseband interface. Hence, all blocks of the transceiver's RF front-end are included in the DUT. The main advantage of this principle, compared to block testing, is the lower effort. There is a very small test overhead that is not depending on the architecture and the technology of the DUT. Therefore the results of the test will not be strongly in- 


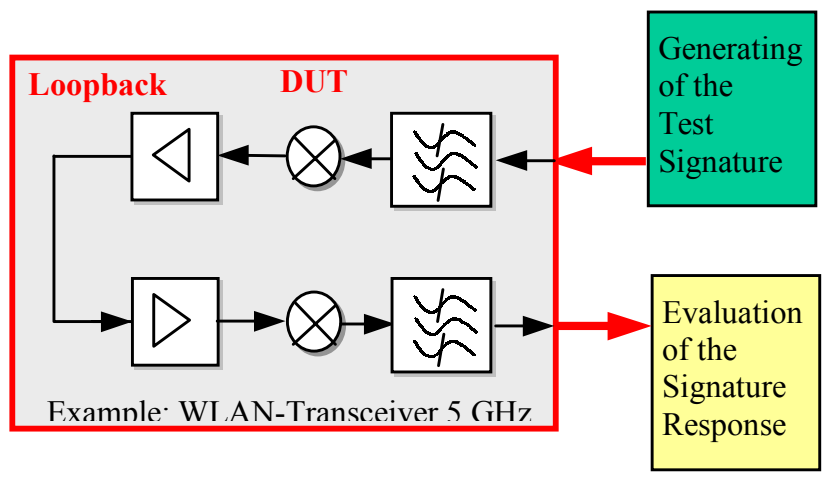

Fig. 1. System testing.

fluenced by failures in the test overhead. Also this principle has a higher flexibility. Adapting the test signature to different requirements can easily carried out by changing the algorithm used for signature generation in the DSP. This allows flexibility in respect of different architectures or technologies (Roberts and Lu, 1995). There exist also disadvantages of this principle. Mainly, the test coverage is lower due to the fact, that the complete transceiver is tested as a whole. Bad spectral properties of the transmitted signal could possible not detected due to a masking effect by the receiver's selectivity. Furthermore, because of the higher complexity of the test signature generation, an additional DSP is required (Mahoney, 1987). In addition both, transmitter and receiver, must be already implemented on silicon.

The argument of low costs for test overhead becomes increasingly important in the case of a quick production test in the high frequency range, such as a $5 \mathrm{GHz}$-system. Extensions towards to implement an optimisation of the signal path using BIST is also possible. In contrast to known concepts for block-orientated self-correction with low operating frequency, the system approach allows to optimise building blocks with high operation frequencies.

\section{Test signature generation and analysis}

Among other approaches test cost reduction is possible by scaling down the quantity of tests and employing low cost testers. In principle such requirements can be fulfilled by applying a complex and optimised test stimulus corresponding to the device under test (DUT). A sufficiently sophisticated post processing system to analyse and evaluate the DUT response is also required.

Testing methodologies of this type are called signature testing. Compared to conventional specification testing, signature testing has the following advantages:

- Multiple DUT specifications can be analysed using a single response acquisition

- Reduced overhead due to the single test stimulus and a single test configuration.

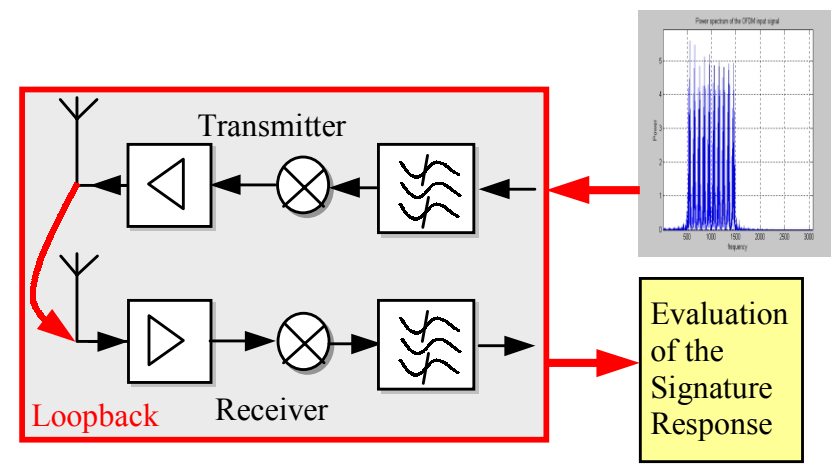

Fig. 2. OFDM test signature.

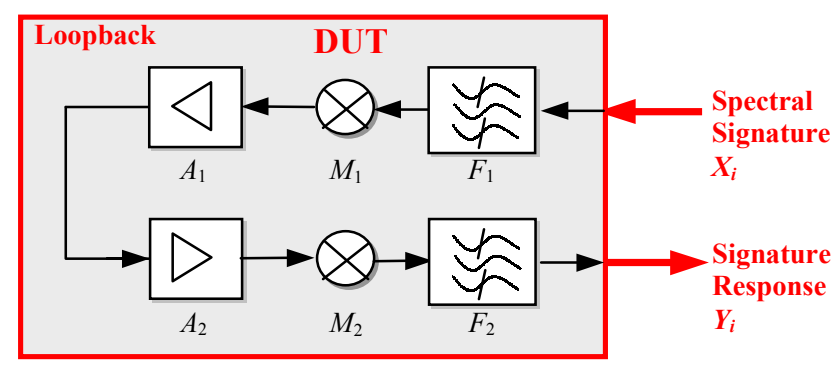

Fig. 3. Chain of stages in a typical architecture.

- Test instruments are less complicated and cheaper.

In the following a multitone OFDM signal (Nee and Prasad, 2000) will be used as spectral test signature. The power spectral density of this signal is depicted in Fig. 2. The motivation for the choice of the OFDM signal results from the fact, that a general characterisation of each block in a RF-FrontEnd is possible by its impulse response. This corresponds to the characterisation of the block by a transfer function in the frequency domain. On the other hand a impulse as a time domain signal would be approximated by a bandwidth-limited noise in frequency domain (Al-Qutayri, 2000). Therefore we propose as the general test signature a multitone OFDM signal.

\subsection{Test signature synthesis}

An approach to generate a time domain test signature that allows the detection of different conventional measured specification parameters is presented in Voorakaranam et al. (2002). Our investigations are focused in particular on WLAN-Front-Ends. Therefore the DUT is a transceiver, and modelling using transfer-functions can be applied. Usually the practical performance of a transceiver is characterised by means of some special parameters like gain, noise figure, IIP3 etc. These parameters are not depending on the specific realisation of the circuit. They are abstract, characterising a block on its behavioural level. In this sense these parameters are closely related to transfer-functions. This underlines our motivation to apply the spectral signature analysis as a 


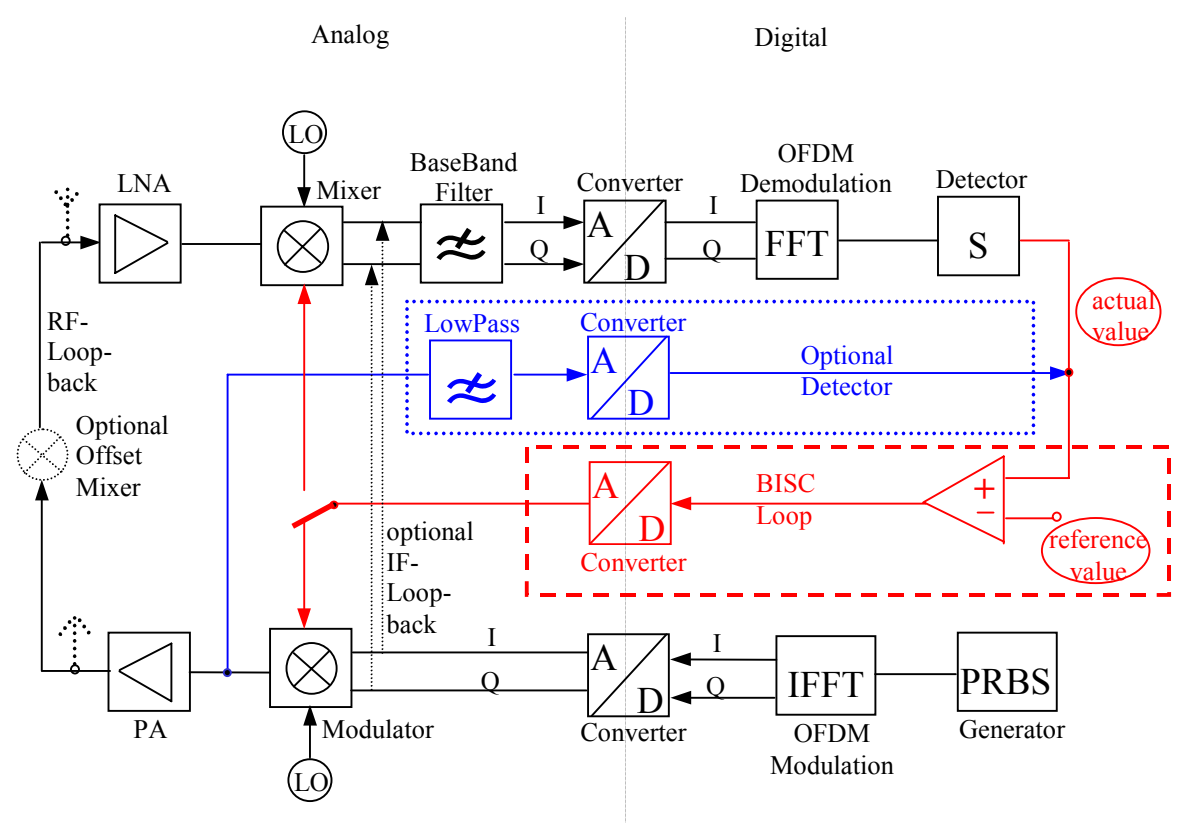

Fig. 4. Block diagram.

suitable BIST-concept and to use an OFDM signal as a test stimulus (Lee et al., 1999).

Let $X(f)$ be the test stimulus. It is of length $n$ and consists of $\mathrm{m}$ discrete spectral lines at frequencies $f_{j}, j=$ $0,1, \ldots, m-1$ different from zero, equidistantly spaced on the frequency axis. We assume $w l$ is the index of the first spectral component $f_{i}$ in $X(f)$ that is unequal zero. Also we assume that $w u=w l+m-1$. Then we get

$X(f)=\left(0, \ldots, 0, X\left(f_{w l}\right), \ldots, X\left(f_{w u}\right), 0, \ldots, 0\right) \equiv$

$\equiv\left(0, \ldots, 0, X_{w l}, \ldots, X_{w u}, 0, \ldots, 0\right)$

Using $X(f)$ as the input signal of the DUT we get the output signal $Y(f)$.

$Y(f)=\left(Y\left(f_{0}\right), \ldots, Y\left(f_{n-1}\right)\right)=\left(Y_{0}, \ldots, Y_{n-1}\right)$

This spectral signature response $Y$ is composed of $n$ spectral lines on discrete frequencies $f_{i}, i=0,1, \ldots, n-1$. Generally it holds $n \geq m$ because of the non linear behaviour of the DUT resulting in intermodulation products. In the case of the spectral signature analysis the property of interest concerns the relation between integrated power at the input and the output of the DUT. That means, the ratio between the input $X_{j}$ at frequency $f_{j}$, and the resulting output $Y_{i}$ at any other frequency $f_{i}$, is of interest. This ratio is known as the transfer factor $T_{i, j}=\frac{Y_{i}}{X_{j}}$. Using transfer factors of this kind gives the possibility of easy modelling the frequency spreading behaviour of a nonlinearity.

This is important for modules of the amplifier type. Regarding the definitions in (1) and (2) we can define a matrix $\mathbf{A}_{\mathbf{k}}$ with transfer factors as matrix elements corresponding to $k^{\text {th }}$ non-linear stage

$\mathbf{A}_{\mathbf{k}}=\left[\begin{array}{ccc}T_{0,0} & \cdots & T_{0, n-1} \\ \vdots & \ddots & \vdots \\ T_{n-1,0} & \cdots & T_{n-1, n-1}\end{array}\right]$

In principle the matrix $\mathbf{A}_{\mathbf{k}}$ describes how the energy is transmitted from the $n^{\text {th }}$ frequency input to the signature response $Y$. It follows that in the case of an ideal and distortionfree amplifier with constant gain over frequency there is no spreading of energy from one frequency to another. Therefore all elements beside of the main diagonal in $\mathbf{A}_{\mathbf{k}}$ are zero.

When the module of interest in the $l^{\text {th }}$ stage is of a nonideal and non-linear mixer type we get the matrix $\mathbf{M}_{\mathbf{l}}$.

$\mathbf{M}_{\mathbf{l}}=\left[\begin{array}{ccc}T_{0,0} & \cdots & T_{0, n-1} \\ \vdots & \ddots & \vdots \\ T_{n-1,0} & \cdots & T_{n-1, n-1}\end{array}\right]$

An ideal mixer with constant conversion gain over frequency and an ideal sideband suppression shifts energy only to the frequency difference between the output frequency of the $l^{\text {th }}$ local oscillator and the discrete frequency of the input signal. Therefore all elements except parallels of the main diagonal are zero.

A different situation is given in the case of a module of filter type. In this case the selectivity of the filter is the most important parameter. It can be easy modelled assuming the filter is linear. In this situation the matrix $\mathbf{F}_{\mathbf{j}}$ can be simplified. Only elements in the main diagonal are non-zero. They corresponds to the complex value of the transfer function of 
signal values at frequencies $f_{0}, \ldots, f_{n-1}$ :

$\mathbf{F}_{\mathbf{j}}=\left[\begin{array}{ccc}T\left(f_{0}\right) & \cdots & 0 \\ \vdots & \ddots & \vdots \\ 0 & \cdots & T\left(f_{n-1}\right)\end{array}\right]$

In practice the behaviour of the filter is non-linear to a certain amount. This situation can be settled modelling the nonlinear filter by a serial of a non-linear amplifier and a linear filter.

The concept of modelling by means of transfer functions could be applied to the whole transceiver (Fig. 3). Exciting the DUT with the spectral signature $X$ the corresponding signature response $Y$ will be formed by all modules included in the chain.

When we assume that the modules of the chain are connected trough non-reactive paths, there exists a simple approach to model the whole chain using the models for the components developed above. In this case it is possible to define the dependencies of the $Y$ from the $X$ by multiplying the matrices. Because of the non-linear properties of the amplifiers and the mixers, the sequence of matrices in the corresponding product is depending on the architecture of the transceiver chain. Therefore it holds:

$Y=f(X)=F_{2} M_{2} A_{2} A_{1} M_{1} F_{1} X$

The expression (6) could be simplified. Since all matrices are of the order $(n, n)$, it is possible to merge all factors

$\mathbf{T}=F_{2} M_{2} A_{2} A_{1} M_{1} F_{1}$

The matrix $\mathbf{T}$ is valid for a defined level of $X$.

Obviously, the frequency domain approach introduced here is advantageous in comparison to a time domain approach described in Voorakaranam et al. (2002). In particular, here is no need to calculate very large matrices like in the time domain approach (Voorakaranam et al., 2002). There the order of the matrices is depending on the number of circuit elements. In our approach only to know the transmission matrices of all stages of the system for the finite number of discrete frequencies is needed. In respect of an optimisation of the signal path extension, only sensitivity matrices of stages corresponding to tuneable parameters are of interest.

\subsection{Test signature response analysis}

The intention of the analysis of the stimulus response consists in the calculation of the so called "distillation quality" of the receiver. That means, the capability of the receiver, to separate wanted signals from all other unwanted signals inside of a chosen frequency band had to be determined.

The wanted components of the spectral signature response inside of the chosen band are determined by

$Y_{w}=\sum_{i=w l}^{w u} Y_{i}$

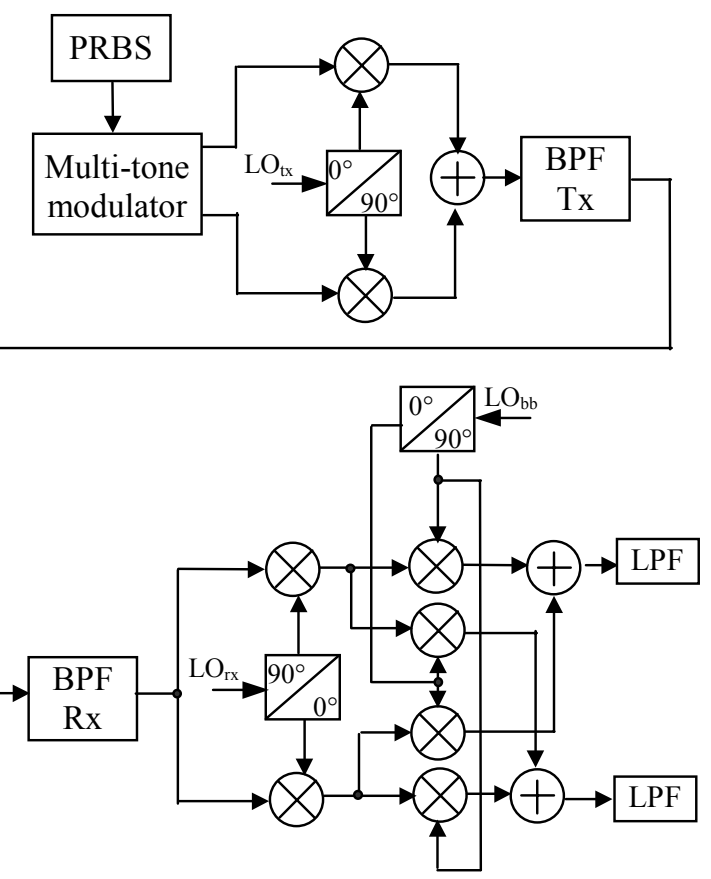

Fig. 5. The simulated transceiver chain.

For the unwanted components of the signature response holds

$Y_{u w}=\sum_{i=0}^{w l-1} Y_{i}+\sum_{i=w u+1}^{n-1} Y_{i}$

For further analysis, the so called global channel selectivity $G C S$ in the frequency domain will be defined. This is the ratio between the power of the wanted signal inside the chosen frequency band $f_{w l} \ldots f_{w u}$ and the sum of the power of the wanted and the unwanted signal:

$G C S=10 \lg \left(\frac{Y_{w}}{Y_{w}+Y_{u w}}\right)$.

Using $G C S$ has two advantages. It takes into account filter behaviour out of tune characterised by slopes of the inband frequency response. In addition, it takes into account interferers and spurs outside the wanted band coming from intermodulations. By uses of $G C S$ the so called disturbance figure $S$ can be determined. It calculates in terms of an I/O relation the degradation of the ratio between wanted and unwanted spectral components. In other terms this means, that the ratio between the global channel selectivity at the input $G C S_{\text {in }}$ and the output $G C S_{\text {out }}$, is calculated, if the test signature passes the DUT.

$S=G C S_{\text {in }}-G C S_{\text {out }}$.

If it is possible to generate a ideal test signature at the baseband input of the transmitter, then, $G C S_{i n}=0 \mathrm{~dB}$. Because of the continuous property of $S$, this figure can very effectively act as the actual value in a control loop necessary for selfcorrection instead of a more "digital" parameter like $B E R$. 
The separation between the wanted and the unwanted spectral components is possible because of the orthogonality of the OFDM carriers. By using the FFT in the OFDM demodulator each carrier will be separated from all others and its amplitude and phase will be determined. This corresponds to the principle of the Fourier Voltmeter (FVM) (Bushnell and Agrawal, 2001; Mahoney, 1987). For CDMA it is shown in Lee et al. (1999), how the measurement of the pilot channel strength as the wanted signal and the total signal strength for calculating SNR, noise figure and other parameters can be carried out. To adopt this principle for OFDM signals requires, that measurement of pilot and total channel strength will be replaced by the measurement of each carrier amplitude using FVM for BIST and BISC purposes.

\section{Aspects of implementation}

We propose the block diagram depicted in Fig. 4 in order to implement the loopback spectral signature analysis for a transceiver front-end. The RF loopback (Nowakowski et al., 2001) is marked by the dotted optional offset mixer. The usage of that mixer depends on the frequency planning of the target application. An attenuator can be also necessary. Beside this, the block diagram depicts a dotted IF loopback. The IF loopback is an additional option. Its application is limited to cases with identical IF in the transmit- ter and the receiver. Using more then one loopback can be reasonable for a self correction process. The optional detection path in the dotted box indicates, that for certain reasons additional effort may be necessary for the detection of the test signature responses. This is especially the case, when the demands in respect of the spectral purity at the transmit antenna are very high and low adjacent channel leakage is required for a transceiver system. In such a case the loopback test of a system of transceiver type is not sufficient in respect of spectral purity. Additional effort must be spent to test and adjust the compatibility with other transceivers not included in the test set-up. The path drawn in the dashed BISC loop box indicates the additional effort for a self-correction control loop. The loopback spectral signature test allows the on-wafer testing for SoC solutions. When the RF front-end is separated from the baseband chip, only on-board testing is possible. In both cases the input test signal can be generated by the baseband chip, if available, or by the tester that emulates the signal generation algorithm of the DSP.

\section{Verification}

To demonstrate the functionality of the proposed method a transceiver chain has been tested. The input signal is a multi carrier signal centred on $325 \mathrm{~Hz}$ with the bandwidth $450 \mathrm{~Hz}$ and a carrier spacing equal to $50 \mathrm{~Hz}$. The input signal is converted up in frequency by an image reject mixer and filtered with a second order Butterworth band-pass filter. The mixer and the filter models the transmitter. The receiver is modelled by another second order band-pass filter, a quadrature

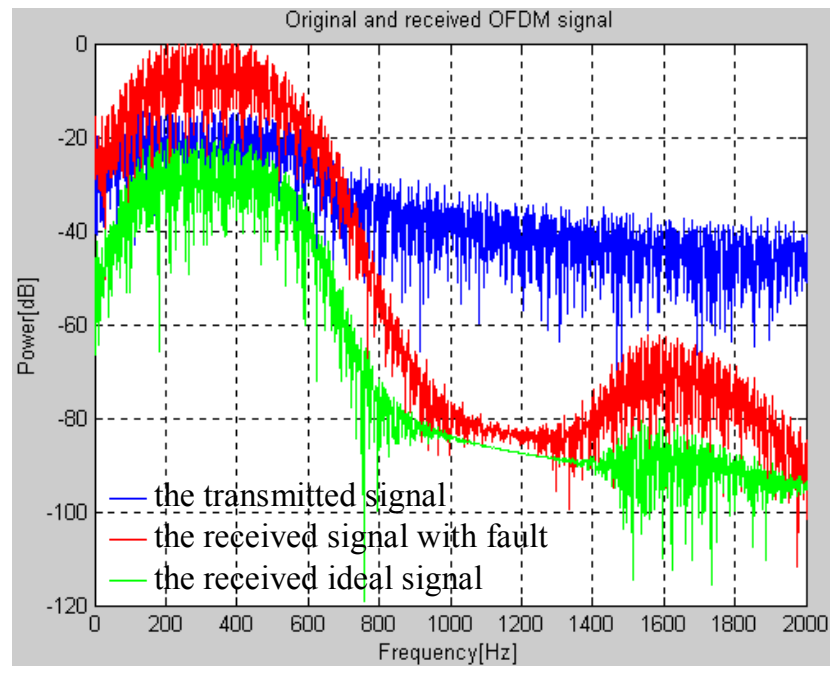

Fig. 6. The input signal, the output signal for linearity error of the band-pass filter and the expected ideal output.

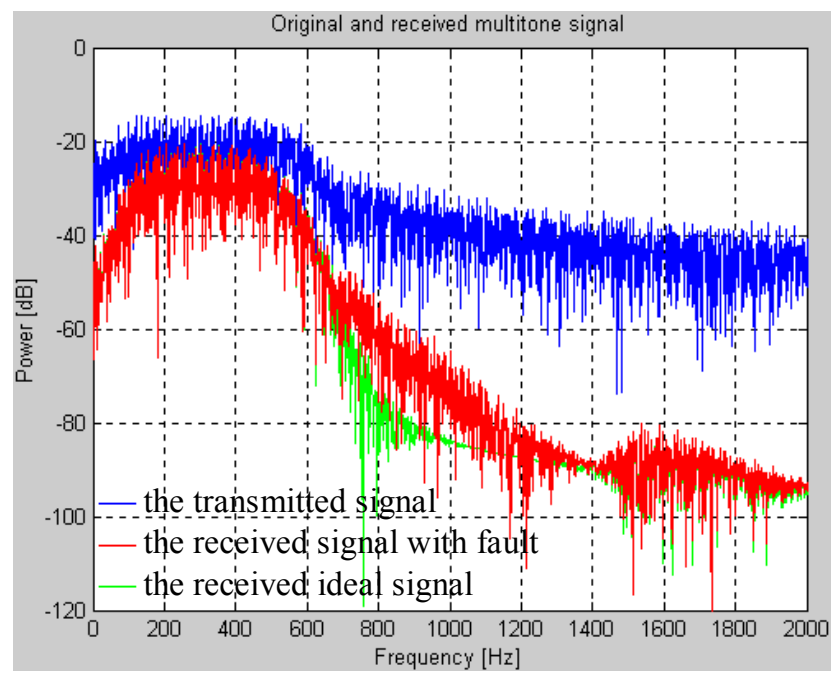

Fig. 7. The input signal, the output signal for phase error of the quadrature mixer and the expected ideal output.

mixer used to generate I and Q paths, a four multiplier image reject mixer and a sixth order Chebyshev low- pass filter for channel selection. The simulated transceiver chain is presented in Fig. 5. Several non-idealities can be introduced in the simulated chain. The most important are non-linearity, amplitude and phase mismatch in the quadrature mixers and frequency errors of the filters. As example the effect of the filter non- linearity on the GCS has been simulated. The power spectral densities of the signals at the input and output of the chain are presented in Fig. 6 . The GCS has been measured against the input signal power. The measured values are given in Table 1.

Another example presents the effect of a non-rejected image signal on the GCS in the quadrature down-converter mixer. The power spectral densities of the signals at the input and output of the chain are presented in Fig. 7. The GCS 
Table 1. GCS variation with the input signal level

\begin{tabular}{cc}
\hline Input power $[\mathrm{dBm}]$ & $G C S[\mathrm{~dB}]$ \\
\hline 5 & -23 \\
2 & -9 \\
1 & -1 \\
0.5 & 6 \\
0.1 & 19 \\
0.05 & 23 \\
0.01 & 17 \\
$5 \times 10^{-3}$ & 12 \\
$1 \times 10^{-3}$ & -1 \\
$5 \times 10^{-4}$ & -7 \\
$1 \times 10^{-4}$ & -21 \\
$5 \times 10^{-5}$ & -27 \\
$1 \times 10^{-5}$ & -35 \\
\hline
\end{tabular}

Table 2. GCS variation with the phase error

\begin{tabular}{cc}
\hline Phase error $\left[^{\circ}\right]$ & $G C S[\mathrm{~dB}]$ \\
\hline$-0.2^{\circ}$ & 33 \\
$-0.1^{\circ}$ & 28 \\
$0^{\circ}$ & 26 \\
$0.1^{\circ}$ & 24 \\
$0.2^{\circ}$ & 23 \\
$0.3^{\circ}$ & 22 \\
$0.4^{\circ}$ & 21 \\
$0.5^{\circ}$ & 20.5 \\
$0.6^{\circ}$ & 20 \\
$0.7^{\circ}$ & 19 \\
$0.8^{\circ}$ & 18.8 \\
$0.9^{\circ}$ & 18.3 \\
\hline
\end{tabular}

variation with the phase error of the image rejection mixer are given in Table 2 .

\section{Conclusions}

In this paper a method called "Spectral Signature Analysis" has been presented. The method consists of two parts: Generation of a test signature and analysis of the signature response of the DUT. This approach allows an optimisation of the signature for self-correction. A block diagram and a simulation environment realised with MATLAB have been presented also. Simulation results show that the proposed method is not only suitable for BIST of RF Front-Ends, but also for BISC.

Acknowledgements. This work has been supported by the German Government (BMBF) under Grant No. 01M3040. In addition, this work has been supported by Nokia Research Centre in Bochum, Infineon AG in Munich and Melexis GmbH in Erfurt.

\section{References}

Bushnell, M. L. and Agrawal, V. D.: Essentials of Electronic Testing for Digital, Memory and Mixed-Signal VLSI Circuits, Boston a.o., Kluwer Acad. Publ. 2nd Ed. 2001.

Mahoney, M.: DSP-Based Testing of Analog and Mixed-Signal Circuits, Washington DC, IEEE Computer Soc. Pr. 1987.

Roberts, G. W. and Lu, A. K.: Analog Signal Generation for BuiltIn-Self-Test of Mixed-Signal Integrated Circuits, Boston a.o., Kluwer Acad. Publ. 1995.

Voorakaranam, R., Cherubal, S., and Chatterjee, A.: A Signature Test Framework for Rapid Production Testing of RF Circuits, Proc. of the Design, Automation and Test in Europe Conference (DATE 2002), Paris, 4-8 March 2002, pp. 186-191, 2002.

Lee, Ch-Y., Panton, W., Granata, G., and Rajkotia, A.: Measurement of Noise Figure, G/T, and $E_{b} / N_{0}$ using RSSI, Proc. of the IEEE MTT-S Symposium on Technologies for Wireless Applications, Anaheim, CA, 17-18 June 1999, pp. 101-103, 1999.

Al-Qutayri, M. A.: System Level Testing of Analog Functions in a Mixed-Signal Circuit, 7th IEEE International Conference on Electronics, Circuits and Systems (ICECS 2000), Beirut, Libanon, 17-20 Dec 2000, Vol. 2, pp. 1026-1029, 2000.

Hafed, M., Abaskharoun, N., and Roberts, G. W.: A Stand-Alone Integrated Test Core for Time and Frequency Domain Measurements, Proc. of the International Test Conference (ITC 2000), Atlantic City, NJ. 3-5 Oct 2000, pp. 1031-1040, 2000.

Hafed, M. M. and Roberts, G. W.: A Stand-Alone Integrated Excitation/Extraction System for Analog BIST Applications, Proc. of the 2000 IEEE Custom Integrated Circuits Conference (CICC'2000), Orlando, FL, 21-24 May 2000, pp. 83-86, 2000.

Nowakowski, J-F., Bonhoure, B., and Carbonero, J. L.: A New Loopback GSM/DCS Bit Error Rate Test Method On Baseband I/Q Outputs, Proc. of the IEEE 57th Automatic RF Techniques Group (ARFTG 57), Phoenix, AZ, 25 May 2001, pp. 113-117, 2001.

Nee, R. v. and Prasad, R.: OFDM Wireless Multimedia Communications, Boston a.o., Artech House, 2000.

Huang, J.-L., Ong, C.-K., and Cheng, K.-T.: A BIST Scheme for On-Chip ADC and DAC Testing, Proc. of the Design, Automation and Test in Europe Conference (DATE 2000), Paris, 27-30 March 2000, pp. 216-220, 2000. 